\title{
Ação de fungos entomopatogênicos em larvas e adultos da mosca do figo Zaprionus indianus (Diptera: Drosophilidae)
}

\author{
Action of entomopathogenic fungi on the larvae and adults of the fig fly Zaprionus indianus \\ (Diptera: Drosophilidae)
}

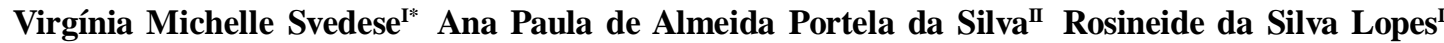 José Ferreira dos Santos ${ }^{\text {III }}$ Elza Áurea de Luna Alves Lima ${ }^{\mathrm{I}}$}

\section{RESUMO}

A mosca do figo, Zaprionus indianus, vem se disseminando no Brasil e causou nos últimos anos perdas de até $50 \%$ na produção de figos. Uma alternativa viável de controle desta mosca pode ser a utilização de fungos entomopatogênicos. Este trabalho foi conduzido $\mathrm{em}$ laboratório $\left(27 \pm 1^{\circ} \mathrm{C}\right.$, UR $70 \pm 10 \%$ e fotoperíodo de $\left.12 \mathrm{~h}\right)$ para avaliar a suscetibilidade dos estágios de larva e adulto de $\mathbf{Z}$. indianus a cinco concentrações $\left(10^{8}\right.$ a $10^{4}$ conídios $\left.m L^{-1}\right)$ de $\boldsymbol{B}$. bassiana (URM2915; ESALQ447) e M. anisopliae (URM3349; URM4403). Não houve mortalidade larval e o período de prépupa não sofreu alteração em relação ao grupo controle, já o estágio de pupa foi aumentado em até três dias quando se utilizou B. bassiana. A emergência de adultos diminuiu em relação ao grupo controle: $10,6 \%$ quando as larvas foram tratadas com a maior concentração de B. bassiana URM2916 e 2,0\% com M. anisopliae URM4403. No bioensaio com adultos, a mortalidade máxima atingiu 98,7\% com B. bassiana e 100,0\% com M. anisopliae. Os menores valores da $C L_{50}$

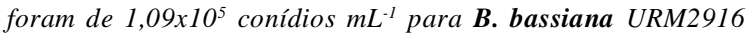
e de $1,94 \times 10^{4}$ conídios $m L^{-1}$ para M. anisopliae URM4403. $O$ tempo letal médio $\left(T L_{50}\right)$ variou de 4,5 a 6,12 dias. Os resultados demonstraram que ambos os fungos são eficientes e mostram ser promissores agentes biocontroladores da mosca do figo, com destaque para M. anisopliae URM4403.

Palavras-chave: Beauveria bassiana, Metarhizium anisopliae, controle biológico, virulência.

\section{ABSTRACT}

The "fig fly", Zaprionus indianus, has spread by in Brazil and in recent years and has caused losses of up to $50 \%$ in the production of figs. A viable alternative to control this fly may be the use of entomopathogenic fungi such. The present study was developed in laboratory $\left(27 \pm 1^{\circ} \mathrm{C}, \mathrm{RH} 70 \pm 10 \%\right.$ and $12 \mathrm{~h}$ photoperiod), to assess the susceptibility of larval and adult stages of $\boldsymbol{Z}$. Indianus to five concentrations $\left(10^{8}\right.$ to $10^{4}$ conidia $\mathrm{ml}^{-1}$ ) of B. bassiana (URM2915; ESALQ447) and M. anisopliae (URM3349; URM4403). There was no larval mortality and the pre-pupal period did not change compared with the control group, whereas the pupal stage was increased by up to three days when we used B. bassiana URM2916. The emergence of adults decreased compared to the control group and was $10.6 \%$ when the larvae were treated with the highest concentration of B. bassiana URM2916 and $2.0 \%$ for $\boldsymbol{M}$. anisopliae URM4403. In the adult's bioassay, the maximum mortality reached $98.7 \%$ with B. bassiana and $100.0 \%$ with $M$. anisopliae. The lowest $L C_{50}$ values were $1.09 \times 105$ conidia $\mathrm{ml}^{-1}$ for B. bassiana URM2916 and $1.94 \times 104$ conidia $\mathrm{ml}^{-1}$ for M. anisopliae URM4403. The medium lethal time (LT50) ranged from 4.5 to 6.12 days. The results showed that both fungi are efficient and promising biocontrol agents of fig fly, mainly M. anisopliae URM4403.

Key words: Beauveria bassiana, Metarhizium anisopliae, biological control, virulence.

\section{INTRODUÇÃO}

A mosca Zaprionus indianus Gupta (Diptera: Drosophilidae), supostamente de origem africana, tem se disseminado nas últimas décadas pelos trópicos, possivelmente pelo aumento do comércio mundial de frutas (VILELA, 1999). O primeiro registro publicado da ocorrência dessa mosca no continente americano ocorreu no Brasil, no Estado de São Paulo,

'Laboratório de Controle Biológico, Universidade Federal de Pernambuco (UFPE), 50670-901, Recife, PE, Brasil. E-mail: vsvedese@ hotmail.com.*Autor para correspondência.

"Biotech ${ }^{\circledR}$ Controle Biológico, Maceió, AL, Brasil.

"IILaboratório de Genética Animal, UFPE. Recife, PE, Brasil. 
onde adultos foram observados se alimentando e fazendo postura em figos (Ficus carica L.) e, por isso, recebeu a denominação de mosca do figo (VILELA, 2001). Depois do primeiro registro, foi encontrada em outras áreas da América do Sul (DE TONI et al., 2001; GOÑI et al., 2001; SANTOS et al., 2003; TIDON et al., 2003), América Central e América do Norte (VAN DER LINDE et al., 2006), mostrando sua grande capacidade de disseminação. Após seu aparecimento no Brasil, devido às condições favoráveis, esta mosca atingiu o status de praga na principal área produtora de figos do Estado de São Paulo, causando perdas na lavoura de até $50 \%$ da produção (AGROFOLHA, 2011).

O uso exclusivo de inseticidas, além de aumentar o custo da produção, inviabiliza a exportação dos frutos e mostra-se ineficaz contra $Z$. indianus, o que exige a adoção de diferentes técnicas de combate dessa praga (RAGA et al., 2006). Uma alternativa viável é o controle biológico utilizando fungos entomopatogênicos, pois eles podem infectar diferentes estágios de desenvolvimento do hospedeiro, como ovos, larvas, pupas e adultos, sendo esta característica desejável e peculiar desse grupo (ONOFRE et al., 2002). Dentre os entomopatógenos, destacam-se Beauveria bassiana (Balsamo) Vuillemin e Metarhizium anisopliae (Metschnikoff) Sorokin que vêm sendo utilizados com sucesso no controle de pragas, tanto no Brasil como em outros países (ALVES, 1998). Trabalhos sobre a ação desses fungos contra diferentes espécies de dípteros mostraram resultados promissores para seu emprego em programas de controle biológico (EKESI et al., 2002; DIMBI et al., 2003; KONSTANTOPOULOU\& MAZOMENOS, 2005; SCHOLTE et al., 2006; COSSENTINE et al., 2010), o que sugere que $\boldsymbol{Z}$. indianus também pode ser suscetível a estes entomopatógenos.

A mosca das frutas, Ceratitis capitata Wiedemann, uma das mais destrutivas pragas da fruticultura mundial, mostrou-se suscetível a $\boldsymbol{B}$. bassiana e a $\boldsymbol{M}$. anisopliae. Os percentuais de mortalidade variaram de 38 a $100 \%$, quando tratadas com B. bassiana, e de 15 a $95 \%$, quando expostas a $\boldsymbol{M}$. anisopliae, demonstrando a variabilidade natural existente entre as linhagens de uma mesma espécie e reforçando a necessidade de bioensaios de patogenicidade para a seleção de linhagens fúngicas mais eficientes (QUESADA-MORAGA, 2006).

Este é o primeiro trabalho que visa a avaliar o efeito dos fungos B. bassiana e M. anisopliae, aplicados em diferentes concentrações de conídios, nas fases de larva e de adulto de $\boldsymbol{Z}$. indianus, de modo a oferecer importantes informações para o controle biológico desta mosca.

\section{MATERIAL E MÉTODOS}

Linhagens fúngicas e hospedeiro utilizado

Foram utilizadas B. bassiana (sensu lato) URM2916 e ESALQ447 (linhagem padrão) e $\boldsymbol{M}$. anisopliae (sensu lato) URM3349 e URM4403. Todas as linhagens foram provenientes da Coleção de Culturas da Micoteca URM, do Departamento de Micologia da Universidade Federal de Pernambuco(UFPE).

A mosca utilizada (Z. indianus) foi coletada em Vila Velha Itamaracá/PE e foram cedidas pelo Laboratório de Genética Animal da UFPE. As moscas são mantidas a aproximadamente $25^{\circ} \mathrm{C}$, em dieta específica para drosofilídeos, à base de banana e extrato de levedura, conforme a metodologia de SANTOS et al. (2003).

\section{Bioensaios}

Para obtenção das suspensões fúngicas, as linhagens foram cultivadas em meio de BatataDextrose-Ágar (BDA) durante 12 dias, sendo, em seguida, inoculadas em arroz cozido e autoclavado, conforme metodologia de VILAS BOAS et al. (1996).

Os bioensaios com larvas e adultos de $\boldsymbol{Z}$. indianus foram realizados em condições controladas de temperatura $\left(26 \pm 1^{\circ} \mathrm{C}\right)$, umidade $(70 \% \pm 10 \mathrm{UR}$ e) e fotofase de $12 \mathrm{~h}$, com seis diferentes tratamentos, sendo cinco formados pelas suspensões fúngicas nas concentrações de $10^{4}, 10^{5}, 10^{6}, 10^{7}, 10^{8}$ conídios $\mathrm{mL}^{-1}$, e um grupo-controle constituído pelo veículo da suspensão de conídios. Para cada tratamento foram feitas três repetições e utilizadas 50 larvas/adultos em cada repetição, totalizando 900 larvas e 900 adultos.

Na infecção larval, grupos de 10 larvas de 3ำ instar foram imersos em $3 \mathrm{~mL}$ das suspensões fúngicas, durante 10 segundos. Em seguida, as larvas foram secas em papel filtro para retirada do excesso da suspensão e transferidas para recipientes transparentes $(10 \mathrm{~cm} \times 5 \mathrm{~cm})$, vedados com espuma e contendo dieta específica para drosofilídeos, nos quais foram colocadas 10 larvas em cada vidro. Foram feitas observações diárias, durante 14 dias, para análise dos seguintes parâmetros biológicos: período de pré-pupa, estágio pupal e percentual de emergência de adultos.

$\mathrm{Na}$ infecção dos adultos, grupos de 10 insetos foram imobilizados durante 10 segundos em éter e pulverizados com pulverizador manual De Vilbiss ${ }^{\circledR} \mathrm{n}^{-} 15$, contendo $1 \mathrm{~mL}$ das suspensões fúngicas. Após o tratamento, as moscas foram mantidas em recipientes iguais aos das larvas com dieta específica. As observações foram feitas diariamente, durante 12 dias, para análise da morte acumulativa.

$\mathrm{O}$ delineamento experimental foi $\mathrm{O}$ inteiramente casualizado para todos os experimentos. Os dados foram submetidos ao teste de normalidade 
de Shapiro-Wilk e à análise de variância, utilizando o teste F (ANOVA) (Programa STATISTIX na versão 7.0) e, em seguida, as médias foram comparadas pelo Teste de Duncan (Programa SAEG na versão 5.0) a 5\% de probabilidade. A análise de Probit foi utilizada para obtenção dos valores da $\mathrm{CL}_{50}$ e do $\mathrm{TL}_{50}$ (após tratamento com a suspensão de $1 \times 10^{8}$ conídios $\mathrm{mL}^{-1}$ ), através do Programa POLO PC.

\section{RESULTADOS E DISCUSSÃO}

Efeito de Beauveria bassiana e Metarhizium anisopliae sobre larvas de Zaprionus indianus
O período de pré-pupa da mosca, a partir de larvas infectadas, variou de um a dois dias quando tratadas com B. bassiana URM2916 e de 1,3 a 1,6 dias com B. bassiana ESALQ447 e não diferiu estatisticamente do grupo controle. Já o estágio de pupa variou de seis a nove dias, sendo que apenas na concentração de $10^{8}$ conídios $\mathrm{mL}^{-1}$ este período diferiu significantemente dos demais tratamentos quando $\boldsymbol{B}$. bassiana URM2916 foi utilizado (Tabela 1). Em experimentos realizados por COSSENTINE et al. (2010), larvas e pupas da mosca da cereja Rhagoletis indifferens Curran (Diptera: Tephritidae) foram expostas a B. bassiana misturada ao solo e os resultados mostraram que cerca de $80 \%$ larvas e $20 \%$

Tabela 1 - Efeito de Beauveria bassiana em parâmetros biológicos do ciclo de vida de Zaprionus indianus, após aplicação de suspensões de conídios, em várias concentrações, nos estágios de larva e adulto.

\begin{tabular}{|c|c|c|c|c|}
\hline \multirow{2}{*}{ Estágios infectados } & \multirow{2}{*}{ Parâmetros biológicos avaliados } & \multirow{2}{*}{$\begin{array}{l}\text { Concentrações das suspensões } \\
\left(\text { conídios } \mathrm{mL}^{-1}\right)\end{array}$} & \multicolumn{2}{|c|}{---Linhagens----- } \\
\hline & & & URM2916 & ESALQ447 \\
\hline \multirow{21}{*}{ Larva } & \multirow{6}{*}{ Pré-pupa (dias) } & $10^{4}$ & $1,06(\mathrm{~A}, \mathrm{a})$ & $1,33(\mathrm{~A}, \mathrm{a})^{1}$ \\
\hline & & $10^{5}$ & $1,46(\mathrm{~A}, \mathrm{a})$ & $1,40(\mathrm{~A}, \mathrm{a})$ \\
\hline & & $10^{6}$ & $1,4(\mathrm{~A}, \mathrm{a})$ & $1,5(\mathrm{~A}, \mathrm{a})$ \\
\hline & & $10^{7}$ & $1,9(\mathrm{~A}, \mathrm{a})$ & $1,5(\mathrm{~A}, \mathrm{a})$ \\
\hline & & $10^{8}$ & $2,0(\mathrm{~A}, \mathrm{a})$ & $1,6(\mathrm{~A}, \mathrm{a})$ \\
\hline & & Controle & $1,0(\mathrm{~A}, \mathrm{a})$ & $1,0(\mathrm{~A}, \mathrm{a})$ \\
\hline & \multirow[t]{3}{*}{$\mathrm{CV}(\%)=14,471$} & & & \\
\hline & & $10^{4}$ & 6,1 (B,a) & 6,1 (B,a) \\
\hline & & $10^{5}$ & $6,2(\mathrm{~B}, \mathrm{a})$ & $6,2(\mathrm{~B}, \mathrm{a})$ \\
\hline & \multirow{3}{*}{ Estágio pupal (dias) } & $10^{6}$ & $6,6(\mathrm{~B}, \mathrm{ab})$ & $6,2(\mathrm{~B}, \mathrm{a})$ \\
\hline & & $10^{7}$ & $6,9(\mathrm{~B}, \mathrm{ab})$ & $6,3(\mathrm{~B}, \mathrm{a})$ \\
\hline & & $10^{8}$ & $9,0(\mathrm{~A}, \mathrm{~b})$ & $8,0(\mathrm{~A}, \mathrm{ab})$ \\
\hline & \multirow{3}{*}{$\mathrm{CV}(\%)=8,273$} & Controle & $6,0(\mathrm{~B}, \mathrm{a})$ & $6,0(\mathrm{~B}, \mathrm{a})$ \\
\hline & & & & \\
\hline & & $10^{4}$ & $57,3(\mathrm{~A}, \mathrm{a})$ & $53,3(\mathrm{~A}, \mathrm{a})$ \\
\hline & \multirow{5}{*}{ Emergência de adultos (\%) } & $10^{5}$ & $51,3(\mathrm{BC}, \mathrm{a})$ & $52,0(\mathrm{~A}, \mathrm{a})$ \\
\hline & & $10^{6}$ & $44,0(\mathrm{C}, \mathrm{a})$ & $39,3(\mathrm{~B}, \mathrm{a})$ \\
\hline & & $10^{7}$ & $40,6(\mathrm{CD}, \mathrm{a})$ & $40,6(\mathrm{~B}, \mathrm{a})$ \\
\hline & & $10^{8}$ & $10,6(D, a)$ & $13,3(\mathrm{C}, \mathrm{a})$ \\
\hline & & Controle & $97,5(\mathrm{E}, \mathrm{a})$ & $97,5(\mathrm{D}, \mathrm{a})$ \\
\hline & $\mathrm{CV}(\%)=10,667$ & & & \\
\hline \multirow{7}{*}{ Adulto } & \multirow{6}{*}{ Mortalidade de adultos (\%) } & $10^{4}$ & $52(\mathrm{~A}, \mathrm{a})$ & $37,3(\mathrm{~A}, \mathrm{~b})$ \\
\hline & & $10^{5}$ & $63,3(\mathrm{~A}, \mathrm{ab})$ & $56,6(\mathrm{~B}, \mathrm{a})$ \\
\hline & & $10^{6}$ & $64(\mathrm{~A}, \mathrm{ab})$ & $68(\mathrm{CD}, \mathrm{a})$ \\
\hline & & $10^{7}$ & $92,66(B, a)$ & $74(\mathrm{D}, \mathrm{b})$ \\
\hline & & $10^{8}$ & $98,66(\mathrm{~B}, \mathrm{a})$ & $88,7(\mathrm{E}, \mathrm{b})$ \\
\hline & & Controle & $9,6(\mathrm{C}, \mathrm{a})$ & $9,6(\mathrm{~F}, \mathrm{a})$ \\
\hline & $\mathrm{CV}(\%)=7,886$ & & & \\
\hline
\end{tabular}

${ }^{(1)}$ Médias seguidas de mesma letra maiúscula na coluna e minúscula na linha, dentro de cada parâmetro avaliado, não diferem entre si pelo teste de Duncan $(\mathrm{P} \geq 0,05)$.

CV: coeficiente de variação

Ciência Rural, v.42, n.11, nov, 2012. 
das pupas foram suscetíveis ao fungo. Por outro lado, larvas e pupas de outras espécies de moscas das frutas, pertencentes à família Tephritidae, não se mostraram suscetíveis a B. bassiana (DE LA ROSA et al., 2002; DIMBI et al., 2003). CASTRILLO et al. (2008) também observaram que este fungo não teve efeito sobre pupas de Scatella tenuicosta Collin (Diptera: Ephydridae). Apesar de algumas linhagens não obterem sucesso na infecção dos estágios iniciais de desenvolvimento dos dípteros, os resultados encontrados revelam que $\boldsymbol{B}$. bassiana URM2916 é potencialmente eficiente para ser usado nestes estágios da mosca do figo.

$\mathrm{O}$ aumento na concentração de $\boldsymbol{B}$. bassiana esteve associado com a diminuição da emergência dos adultos, e este resultado foi observado nas duas linhagens (Tabela 1). O percentual médio de emergência das moscas após 14 dias variou de 57,3 a 10,6\% quando expostas à B. bassiana URM2916 e de 53,3 a 13,3\% com B. bassiana ESALQ447, não apresentando diferença estatística significativa entre as linhagens testadas. ALMEIDA et al. (2007) demonstraram que a emergência de adultos de $\boldsymbol{C}$. capitata foi reduzida a $6,4 \%$ quando tratadas com $5 \times 10^{8}$ conídios $\mathrm{mL}^{-1}$ de $\boldsymbol{B}$. bassiana, contudo ao utilizarem concentrações menores, a emergência não diferiu do controle.

Quando as larvas foram infectadas com $\boldsymbol{M}$. anisopliae, o período de pré-pupa ficou entre 1,12 e 2,02 dias sem diferença significativa em relação ao grupo controle (1,3 dias) e entre as concentrações utilizadas. As linhagens de $\boldsymbol{M}$. anisopliae utilizadas no presente estudo também não causaram mudanças significativas no estágio pupal (Tabela 2). Embora o fungo não tenha agido nesses estágios, de acordo com DARWISH \& ZAYED (2002), os efeitos da infecção em larvas podem ser demonstrados em estágios posteriores, como na redução da emergência de adultos.

Como se observa na tabela 2 , as duas linhagens de $\boldsymbol{M}$. anisopliae reduziram significativamente a emergência de adultos de $\boldsymbol{Z}$. indianus, em relação ao grupo controle, não sendo observadas diferenças estatísticas entre as linhagens, exceto na concentração de $10^{4}$ conídios $\mathrm{mL}^{-1}$. Na concentração de $1 \times 10^{8}$ conídios $\mathrm{mL}^{-1}$, menos de $3 \%$ dos insetos emergiram, confirmando assim a potencialidade das duas linhagens para o controle das larvas de $\boldsymbol{Z}$. indianus. Estudos em laboratório mostraram que uma alta concentração de conídios $\left(1 \times 10^{8}\right.$ conídios $\left.\mathrm{mL}^{-1}\right)$ de $\boldsymbol{M}$. anisopliae é necessária para diminuir a emergência de $\boldsymbol{C}$. capitata (ALVES et al., 2004).

Ação de Beauveria bassiana e Metarhizium anisopliae sobre adultos de Zaprionus indianus

A mortalidade variou de 52 a $98,66 \%$, quando tratados com B. bassiana URM2916, e de 37,3 a $88,7 \%$, quando se utilizou B. bassiana ESALQ447, sendo que a mortalidade do grupo controle não excedeu $10 \%$ (Tabela 1). Houve diferença significativa entre as linhagens na maioria das concentrações utilizadas, em que B. bassiana URM2916 causou mortalidade superior a B. bassiana ESALQ447. KONSTANTOPOULOU \& MAZOMENOS (2005) testaram a virulência de B. bassiana e de Beauveria brongniartii (Saccardo) Petch sobre adultos da mosca da oliveira Bactrocera oleae Gmelin (Diptera: Tephritidae) e $\boldsymbol{C}$. capitata. Os bioensaios revelaram que apenas B. bassiana causou alta mortalidade de $\boldsymbol{B}$. oleae, contudo $\boldsymbol{C}$. capitata foi suscetível as duas espécies de Beauveria. MAHMOUD (2009) também avaliou a ação de $\boldsymbol{B}$. bassiana e $\boldsymbol{M}$. anisopliae contra adultos de $\boldsymbol{B}$. oleae sob condições de laboratório. A mortalidade causada por esses fungos, após 20 dias de tratamento, foi de 60,8 e $39,2 \%$, respectivamente.

Quando os adultos foram tratados com $\boldsymbol{M}$. anisoplae URM3349, a mortalidade variou de 40,67 a $100 \%$ e não houve diferença estatística entre as concentrações a partir de $10^{7}$ conídios $\mathrm{mL}^{-1}$; quando tratados com M. anisopliae URM4403 a mortalidade variou de 52,67 a 100\%, sendo esta última linhagem a que causou as maiores taxas de mortalidade nas diferentes concentrações (Tabela 2). Resultados promissores foram obtidos por MIGIRO et al. (2010), ao estudarem o efeito de $\boldsymbol{M}$. anisopliae sobre adultos de Liriomyza huidobrensis Blanchard (Diptera: Agromyzidae), díptero responsável por grandes perdas na produção de hortaliças. Os autores observaram que a melhor linhagem foi capaz de causar $100 \%$ de mortalidade, sugerindo o uso deste fungo em programas de controle biológico desta praga.

O valor da $\mathrm{CL}_{50}$ para B. bassiana $\mathrm{URM} 2916$ foi de $1,09 \times 10^{5}$ e de $3,8 \times 10^{6}$ conídios $\mathrm{mL}^{-1}$ para B. bassiana ESALQ447, confirmando que a primeira linhagem foi mais virulenta. Já para M. anisopliae URM3349 a CL $\mathrm{L}_{50}$ foi de $1,64 \times 10^{5}$ conídios $\mathrm{mL}^{-1}$ e de $1,94 \times 10^{4}$ conídios $\mathrm{mL}^{-1}$ para M. anisopliae URM3349 (Tabela 3). Os valores de $\chi^{2}$ revelaram a homogeneidade dos dados para a equação de regressão e, considerando os valores entre os intervalos de confiança, $\boldsymbol{Z}$. indianus foi suscetível às linhagens testadas, porém a linhagem $\boldsymbol{M}$. anisopliae URM4403 foi mais efetiva, apresentando menor $\mathrm{CL}_{50}$.

As diferenças na virulência entre as linhagens de $\boldsymbol{B}$. bassiana foram confirmadas pelos valores do $\mathrm{TL}_{50}$. Na concentração de $10^{8}$ conídios $\mathrm{mL}^{-1}$, o $\mathrm{TL}_{50}$ foi de 5,3 dias para B. bassiana URM2916 e de 6,1 dias para B. bassiana ESALQ447 (Tabela 4). Em laboratório, formulações contendo elevadas concentrações de conídios $\left(10^{9}\right.$ conídios $\left.\mathrm{g}^{-1}\right)$ de $\boldsymbol{B}$. bassiana provocaram mortalidade de $98,7 \%$ de adultos de Anastrepha ludens (Loew), apresentando um TL To $_{50}$ 
Tabela 2 - Efeito de Metarhizium anisopliae em parâmetros biológicos do ciclo de vida de Zaprionus indianus, após aplicação de suspensões de conídios, em várias concentrações, nos estágios de larva e adulto.

\begin{tabular}{|c|c|c|c|c|}
\hline \multirow{2}{*}{ Estágios infectados } & \multirow{2}{*}{ Parâmetros biológicos avaliados } & \multirow{2}{*}{$\begin{array}{l}\text { Concentrações das suspensões } \\
\quad\left(\text { conídios } \mathrm{mL}^{-1}\right)\end{array}$} & \multicolumn{2}{|c|}{ Linhagens } \\
\hline & & & URM3349 & URM4403 \\
\hline \multirow{21}{*}{ Larva } & \multirow{5}{*}{ Pré-pupa (dias) } & $10^{4}$ & $1,68(\mathrm{~A}, \mathrm{a})^{1}$ & $1,12(\mathrm{~A}, \mathrm{a})$ \\
\hline & & $10^{5}$ & $1,60(\mathrm{~A}, \mathrm{a})$ & $1,23(\mathrm{~A}, \mathrm{a})$ \\
\hline & & $10^{6}$ & $1,78(\mathrm{~A}, \mathrm{a})$ & $1,33(\mathrm{~A}, \mathrm{a})$ \\
\hline & & $10^{7}$ & $1,70(\mathrm{~A}, \mathrm{a})$ & $1,53(\mathrm{~A}, \mathrm{a})$ \\
\hline & & $10^{8}$ & $2,02(\mathrm{~A}, \mathrm{a})$ & $1,50(\mathrm{~A}, \mathrm{a})$ \\
\hline & \multirow{3}{*}{$\mathrm{CV}(\%)=14,395$} & Controle & $1,30(\mathrm{~A}, \mathrm{a})$ & $1,30(\mathrm{~A}, \mathrm{a})$ \\
\hline & & & & \\
\hline & & $10^{4}$ & $5,22(\mathrm{~A}, \mathrm{a})$ & $5,27(\mathrm{~A}, \mathrm{a})$ \\
\hline & \multirow{4}{*}{ Estágio pupal (dias) } & $10^{5}$ & $5,32(\mathrm{~A}, \mathrm{a})$ & $5,47(\mathrm{~A}, \mathrm{a})$ \\
\hline & & $10^{6}$ & $5,58(\mathrm{~A}, \mathrm{a})$ & $6,20(\mathrm{~A}, \mathrm{a})$ \\
\hline & & $10^{7}$ & $5,77(\mathrm{~A}, \mathrm{a})$ & $6,27(\mathrm{~A}, \mathrm{a})$ \\
\hline & & $10^{8}$ & $6(\mathrm{~A}, \mathrm{a})$ & $6,33(\mathrm{~A}, \mathrm{a})$ \\
\hline & \multirow{4}{*}{$\mathrm{CV}(\%)=10,363$} & Controle & $5,93(\mathrm{~A}, \mathrm{a})$ & $5,93(\mathrm{~A}, \mathrm{a})$ \\
\hline & & & & \\
\hline & & $10^{4}$ & $70,67(\mathrm{C}, \mathrm{a})$ & $54(\mathrm{D}, \mathrm{b})$ \\
\hline & & $10^{5}$ & $42,67(B, a)$ & $46,67(\mathrm{CD}, \mathrm{a})$ \\
\hline & \multirow{3}{*}{ Emergência de adultos (\%) } & $10^{6}$ & $13,33(\mathrm{~A}, \mathrm{ab})$ & $31,33(\mathrm{C}, \mathrm{a})$ \\
\hline & & $10^{7}$ & $14,67(\mathrm{~A}, \mathrm{ab})$ & $18,00(\mathrm{~B}, \mathrm{a})$ \\
\hline & & $10^{8}$ & $2,67(\mathrm{~A}, \mathrm{a})$ & $2,00(\mathrm{~A}, \mathrm{a})$ \\
\hline & & Controle & $97,33(\mathrm{D}, \mathrm{a})$ & $97,33(\mathrm{E}, \mathrm{a})$ \\
\hline & $\mathrm{CV}(\%)=8,668$ & & & \\
\hline \multirow{7}{*}{ Adulto } & \multirow{6}{*}{ Mortalidade de adultos (\%) } & $10^{4}$ & $40,67(\mathrm{~B}, \mathrm{a})$ & $52,67(\mathrm{C}, \mathrm{a})$ \\
\hline & & $10^{5}$ & $55,33(\mathrm{~B}, \mathrm{a})$ & $71,33(\mathrm{~B}, \mathrm{a})$ \\
\hline & & $10^{6}$ & $59,33(\mathrm{~B}, \mathrm{a})$ & $86,67(\mathrm{AB}, \mathrm{a})$ \\
\hline & & $10^{7}$ & $83,33(\mathrm{~A}, \mathrm{a})$ & $100(\mathrm{~A}, \mathrm{a})$ \\
\hline & & $10^{8}$ & $100(\mathrm{~A}, \mathrm{a})$ & $100(\mathrm{~A}, \mathrm{a})$ \\
\hline & & Controle & $10,67(\mathrm{C}, \mathrm{a})$ & $10,67(\mathrm{D}, \mathrm{a})$ \\
\hline & $\mathrm{CV}(\%)=12,888$ & & & \\
\hline
\end{tabular}

${ }^{(1)}$ Médias seguidas de mesma letra maiúscula na coluna e letras minúscula na linha, dentro de cada parâmetro avaliado, não diferem entre si, pelo teste de Duncan $(\mathrm{P} \geq 0,05)$.

$\mathrm{CV}$ : coeficiente de variação.

Tabela 3 - Concentração letal $\left(\mathrm{CL}_{50}\right)$ (conídios $\mathrm{mL}^{-1}$ ) das linhagens de Beauveria bassiana (URM2916 e Esalq447) e Metarhizium anisopliae (URM3349 e URM4403) aplicadas em adultos de Zaprionus indianus

\begin{tabular}{llll}
\hline Linhagens & \multicolumn{1}{c}{$\mathrm{CL}_{50}(\mathrm{IC})^{(\mathrm{a})}$} & \multicolumn{1}{c}{ Equação de regressão } & $\chi^{2(\mathrm{~b})}$ \\
\hline URM2916 & $1,09 \times 10^{5}\left(0,28 \times 10^{4}-6,6 \times 10^{5}\right)$ & $\mathrm{Y}=2,785+0,4396 * \log \mathrm{x}$ & 5,13 \\
ESALQ447 & $3,8 \times 10^{6}\left(1,4 \times 10^{5}-, 8 \times 10^{7}\right)$ & $\mathrm{Y}=1,3934+0,5477 * \log \mathrm{x}$ & 6,19 \\
URM3349 & $1,64 \times 10^{5}\left(0,19 \times 10^{4}-1,14 \times 10^{6}\right)$ & $\mathrm{Y}=2,198+0,537 * \log \mathrm{x}$ & 8,01 \\
URM4403 & $1,94 \times 10^{4}\left(0,67 \times 10^{4}-0,41 \times 10^{5}\right)$ & $\mathrm{Y}=1,914+0,719 * \log \mathrm{x}$ & 2,90 \\
\hline
\end{tabular}

(a) Intervalo de confiança a $95 \%$.

(b) Calculado pelo Probit. 
Tabela 4 - Tempo letal médio (TL ${ }_{50}$ ) (dias) das linhagens de Beauveria bassiana (URM2916 e ESALQ447) e Metarhizium anisopliae (URM3349 e URM4403) após aplicação de $1 \times 10^{8}$ conídios $\mathrm{mL}^{-1}$ em adultos de Zaprionus indianus.

\begin{tabular}{llcl}
\hline Linhagens & $\mathrm{TL}_{50} \mathrm{e} \mathrm{IC}^{(\mathrm{a})}(\mathrm{dias})$ & Equação de regressão & $\chi^{2(\mathrm{~b})}$ \\
\hline URM2916 & $5,33(4,80-5,84)$ & $1,3218+5,061 * \log \mathrm{X}$ & 17,95 \\
ESALQ447 & $6,12(5,51-6,45)$ & $1,8529+4,113 * \log \mathrm{X}$ & 26,7 \\
URM3349 & $5,5(5,8-6,74)$ & $1,2129+3,881 * \log \mathrm{X}$ & 26,2 \\
URM4403 & $4,5(3,8-5,3)$ & $2,6535+2,310 * \log \mathrm{X}$ & 15,9 \\
\hline
\end{tabular}

(a) Intervalo de confiança a $95 \%$.

(b) Calculado pelo Probit

de 4,2 dias (TOLEDO et al., 2007). Para M. anisopliae URM3349, o TL ${ }_{50}$ foi de 5,5 dias, já M. anisopliae URM4403 apresentou tempo letal de 4,5 dias (Tabela 4), demonstrando que esta última linhagem foi a mais eficiente. Diferenças entre os tempos letais é uma ferramenta bastante utilizada na seleção de linhagens, pois é interessante que o fungo mate rapidamente seus hospedeiros (LOHMEYER \& MILLER, 2006).

\section{CONCLUSÃO}

Os fungos B. bassiana e M. anisopliae afetam negativamente o desenvolvimento da mosca do figo. As linhagens avaliadas são potencialmente virulentas aos adultos de $\boldsymbol{Z}$. indianus, causando elevadas taxas de mortalidade, principalmente nas maiores concentrações, destacando $\boldsymbol{M}$. anisopliae URM4403 e B. bassiana URM2916 como as mais eficientes, pois além da alta mortalidade apresentam $\mathrm{CL}_{50}$ e $\mathrm{TL}_{50}$ menores. Esses fungos possuem potencial para utilização em programas de controle biológico da mosca do figo.

\section{AGRADECIMENTOS}

Os autores agradecem ao Conselho Nacional de Desenvolvimento Científico e Tecnológico ( $\mathrm{CNPq}$ ) e ao Banco do Nordeste do Brasil (BNB), pelo suporte financeiro, e ao Dr. Venézio dos Santos, pelo auxílio nas análises estatísticas.

\section{REFERÊNCIAS}

AGROFOLHA. Mosca-do-figo traz prejuízo de $\mathbf{R} \$ 2$ milhões em Valinhos. Disponível em: <http://www.todafruta.com.br/ portal/icaNoticiaAberta.asp?idNoticia $=1547>$. Acesso em: 14 jul. 2011

ALMEIDA, J.E.M. et al. Pathogenicity of the entomopathogenic fungi and nematode on medfly Ceratitis capitata (Wied.) (Diptera: Tephritidae). BioAssay, v.2, p.17, 2007. Disponível em: 〈http://www.bioassay.org.br/vol2.php〉. Acesso em: 10 abr. 2011.
ALVES, S.B. Fungos entomopatogênicos. In: ALVES, S.B. Controle microbiano de insetos. Piracicaba: FEALQ, 1998. Cap.11, p.289-381.

ALVES, S.B. et al. Avaliação de fungos entomopatogênicos para Ceratitis capitata. Manejo Integrado de Plagas y Agroecologia, v.72, p.31-38, 2004. Disponível em: <http://www.scielo.unal.edu.co/ scielo.php?script=sci_arttext\&pid=S0120-04882010000200006 \&lng=pt\&nrm=iso>. Acesso em: 21 ago. 2011.

CASTRILLO, L.A. et al. Molecular characterization and comparative virulence of Beauveria bassiana isolates (Ascomycota: Hypocreales) associated with the greenhouse shore fly, Scatella tenuicosta (Diptera: Ephydridae). Biological Control, v.45, p.154-162, 2008. Disponível em: <http:// ddr.nal.usda.gov/bitstream/.../IND44029437.pdf>. Acesso em: 07 jun. 2011. doi:10.1016/j.biocontrol.2007.10.010.

COSSENTINE, J. et al. Susceptibility of preimaginal western cherry fruit fly, Rhagoletis indifferens (Diptera: Tephritidae) to Beauveria bassiana (Balsamo) Vuillemin Clavicipitaceae (Hypocreales). Journal of Invertebrate Pathology, v.104, p.105-109, 2010. Disponível em: <http://www.bioone.org/doi/ abs/10.1603/0022-0493-99.6.1955? journalCode=ecen>. Acesso em: 12 nov. 2011. doi: 10.1603/0022-0493-99.6.1955.

DARWISH, E.; ZAYED, A. Pathogenicity of two entomopathogenic hyphomycetes, Beauveria bassiana and Metarhizium anisopliae, to the housefly Musca domestica. Journal of the Egyptian Society of Parasitology, v.32, p.785-796, 2002. Disponível em: <http://www.mendeley.com/ research/selective-isolation-of-the-entomopa thogenic-fungibeauveria-bassiana-and-metarhizium-anisopliae-from-anartificial-potting-medium/>. Acesso em: 05 set. 2011.

DE LA ROSA, W. et al. Beauveria bassiana as a pathogen of the Mexican fruit fly (Diptera: Tephritidae) under laboratory conditions. Journal of Economic Entomology, v.95, p.3643, 2002. Disponível em: <http://www.bioone.org/doi/abs/ 10.1603/0022-0493-95.1.36? journalCode=ecen $>$. Acesso em: 31 jan. 2012. doi: http://dx.doi.org/10.1603/0022-0493-95.1.36.

DE TONI, D.C. et al. First register of Zaprionus indianus (Diptera: Drosophilidae) in the state of Santa Catarina. Biotemas, v.14, p.71-85, 2001. Disponível em: <http:// www.drosofilideos.ufsc.br/artigos/zaprionusind.pdf >. Acesso em: 15 jun. 2011.

DIMBI, S.P. et al. Pathogenicity of Metarhizium anisopliae (Metsch.) Sorokin and Beauveria bassiana (Balsamo) Vuillemin, 
to three adult fruit fly species: Ceratitis capitata (Weidemann), C. rosa var. fasciventris Karsch and C. cosyra (Walker) (Diptera: Tephritidae). Mycopathologia, v.156, p.375-382, 2003. Disponível em: <http://www.springerlink.com/content/ v575071254702645/>. Acesso em: 27 set. 2011. doi: 10.1023/ B:MYCO.0000003579.48647.16.

EKESI, S. et al. Mortality in three african tephritid fruit fly puparia and adults caused by the entomopathogenic fungi, Metarhizium anisopliae and Beauveria bassiana. Biocontrol Science and Technology, v.12, p.7-17, 2002. Disponível em: <http://pubget.com/search?q=issn\%3A0958-3157+vol\%3A12 +issue\%3A1\&from=pgtmp_17bc426c67a84341f3659c688b0cb2ec >. Acesso em: 28 mar. 2011. doi: 10.1080/09583150120093077.

GOÑI, B. et al. First record of Zaprionus indianus Gupta, 1970 (Diptera: Drosophilidae) in southern localities of Uruguay. Drosophila Information Service, v.84, p.61-64, 2001. Disponível em: <http://www.ou.edu/journals/dis/DIS84/ index.html>. Acesso em: 19 ago. 2011.

KONSTANTOPOULOU, M.A.; MAZOMENOS, B.E. Evaluation of Beauveria bassiana and B. brongniartii strains and four wild-type fungal species against adults of Bactrocera oleae and Ceratitis capitata. BioControl, v.50, p.293-305, 2005. Disponível em: <http://www.mendeley.com/research/evaluationbeauveria-bassiana-b-brongniartii-strains-wildtype-fungal-speciesadults-bactrocera-oleae-ceratitis-capitata-3/>. Acesso em: 13 jul. 2011. doi: doi.org/10.1007/s10526-004-0458-4.

LOHMEYER, K.H.; MILLER, J.A. Pathogenicity of three formulations of entomopathogenic fungi for control of adult Haematobia irritans (Diptera: Muscidae). Journal of Economic Entomology, v.99, p.1943-1947, 2006. Disponível em: <http://www.bioone.org/doi/abs/10.1603/0022-049399.6.1943>. Acesso em: 13 jul. 2011. doi: http://dx.doi.org/ 10.1603/0022-0493-99.6.1943.

MAHMOUD, F.M. Pathogenicity of three commercial products of entomopathogenic fungi, Beauveria bassiana, Metarhizum anisopilae and Lecanicillium lecanii against adults of olive fly, Bactrocera oleae (Gmelin) (Diptera: Tephritidae) in the laboratory. Plant Protection Science, v.45, p.98-102, 2009. Disponível em: <http://www.agriculturejournals.cz/web/ pps.htm? volume $=45 \&$ type $=$ volume $>$. Acesso em: 21 ago. 2011 .

MIGIRO, L.N. et al. Pathogenicity of entomopathogenic fungi Metarhizium anisopliae and Beauveria bassiana (Hypocreales: Clavicipitaceae) isolates to the adult pea leafminer (Diptera: Agromyzidae) and prospects of an autoinoculation device for infection in the field. Environmental Entomology, v.39, p.468-75, 2010. Disponível em: <http://www.bioone.org/ doi/pdf/10.1603/EN09359>. Acesso em: 05 out. 2011. doi: 10.1603/EN09359.

ONOFRE, S.B. et al. Controle biológico de pragas na agropecuária por meio de fungos entomopatogênicos. In. SERAFINI, L.A. et al. Biotecnologia: avanços na agricultura e na agroindústria. Caxias do Sul: EDUCS, 2002. Cap.8, p.295-328.

QUESADA-MORAGA, E. et al. Laboratory evaluation of entomopathogenic fungi Beauveria bassiana and Metarhizium anisopliae against puparia and adults of Ceratitis capitata (Diptera: Tephritidae). Journal of Economic Entomology, v.99, p.19551966, 2006. Disponível em: <http://www.bioone.org/doi/abs/
10.1603/0022-0493-99.6.1955? journalCode=ecen>. Acesso em: 08 set. 2011. doi: http://dx.doi.org/10.1603/0022-0493-99.6.1955.

RAGA, A. et al. Eficácia de atrativos alimentares na captura de moscas-das-frutas em pomar de citros. Bragantia, v.65, p.337345, 2006. Disponível em: <http://www.scielo.br/

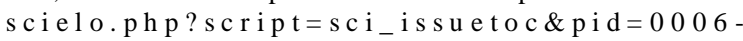
$870520060002 \& \operatorname{lng}=$ pt\&nrm=iso $>$. Acesso em: 15 out. 2011.

SANTOS, J.F. et al. Colonization of northeast region of Brazil by the drosophilid flies Drosophila malerkotliana and Zaprionus indianus, a new potential pest for Brazilian fruit culture. Drosophila Information Service, v.86, p.92-95, 2003. Disponível em: <http://www.ou.edu/journals/dis/DIS84/ index.html>. Acesso em: 15 out. 2011.

SCHOLTE, E.J. et al. Infection of the malaria mosquito Anopheles gambiae with the entomopathogenic fungus Metarhizium anisopliae reduces blood feeding and fecundity. Journal of Invertebrate Pathology, v.91, p.43-49, 2006. Disponível em: <http://www.sciencedirect.com/science/article/ pii/S002220110500217X>. Acesso em: 10 set. 2011. doi: http://dx.doi.org/10.1016/j.jip.2005.10.006.

TIDON, R. et al. Impact of the colonization of Zaprionus (Diptera: Drosophilidae) indifferent ecosystems of the Neotropical Region: 2 years after the invasion. Biological Conservation, v.112, p.299-305, 2003. Disponível em: <http://www.sciencedirect.com/science/journal/00063207/112/ 3>. Acesso em: 05 out. 2011. doi: http://dx.doi.org/10.1016/ S0006-3207(02)00322-1.

TOLEDO, J. et al. Horizontal transmission of Beauveria bassiana in Anastrepha ludens (Diptera: Tephritidae) under laboratory and field cage conditions. Journal of Economic Entomology, v.100, p.291-297, 2007. Disponível em: <http://www.bioone.org/ doi/abs/10.1603/00220493(2007)100\%5B291: HTOBBI\%5D2.0.CO\%3B2>. Acesso em: 15 nov. 2011. doi: http://dx.doi.org/10.1603/0022-0493(2007)100[291: HTOBBI]2.0.CO;2.

VAN DER LINDE, K. et al. First records of Zaprionus indianus (Diptera: Drosophilidae), a pest species on commercial fruits from Panama and the United States of America. Florida Entomologist, v.89, p.402-404, 2006. Disponível em: <http://www.bioone.org/ doi/abs/10.1603/00220493\%282007\%29100\% 5B291:HTOBBI\%5D2.0.CO\%3B2>. Acesso em: 11 out. 2011. doi:http://dx.doi.org/10.1603/0022-0493(2007)100[291: HTOBBI]2.0.CO;2.

VILAS BOAS, A.M. et al. Diversificação de meios de cultura para produção de fungos entomopatogênicos. Arquivo de Biologia e Tecnologia, v.39, p.123-128, 1996. Disponível em: <http:// www.scielo.br/scielo.php? script $=$ sci_issuetoc\&pid $=$ 1516891320010004\&lng=en\&nrm=iso>. Acesso em: 09 ago. 2011.

VILELA, C.R. Is Zaprionus indianus Gupta, 1970 (Diptera: Drosophilidae) currently colonizing the Neotropical Region? Drosophila Information Service, v.82, p.37-39. 1999. Disponível em: <http://www.ou.edu/journals/dis/>. Acesso em: 23 set. 2011.

VILELA, C.R. et al. Mosca-africana-do-figo, Zaprionus indianus (Diptera: Drosophilidae). In: Histórico e impacto das pragas introduzidas no Brasil. Ribeirão Preto: Holos, 2001. Cap.4, p.48-51. 